Int. Journal of Math. Analysis, Vol. 7, 2013, no. 1, 31 - 42

\title{
Existence of Positive Solutions and Iterative Approximations for a Second Order Neutral Delay Differential Equation
}

\author{
Zeqing Liu \\ Department of Mathematics \\ Liaoning Normal University \\ Dalian, Liaoning 116029, People's Republic of China \\ zeqingliu@163.com \\ Jingjing Zhu \\ Department of Mathematics \\ Liaoning Normal University \\ Dalian, Liaoning 116029, People's Republic of China \\ jingjingzhu2@126.com \\ Shin Min Kang* \\ Department of Mathematics and RINS \\ Gyeongsang National University \\ Jinju 660-701, Korea \\ smkang@gnu.ac.kr \\ ${ }^{*}$ Corresponding author
}

\begin{abstract}
The paper is concerned with the existence of positive solutions and convergence of Mann iterative methods for a second order neutral delay differential equation. Three nontrivial examples are presented.
\end{abstract}

Mathematics Subject Classification: 34K40

Keywords: Second order neutral delay differential equation, uncountably many bounded positive solutions, contraction mapping, Mann iterative method. 


\section{Introduction and Preliminaries}

Recently, some researchers discussed the existence of solutions for the first and second order neutral delay differential equations, for example, see [1]-[4] and the references cited therein. Zhang et al. [3] established the existence of a nonoscillatory solution for the first order linear neutral differential equation with positive and negative coefficients

$$
[x(t)+p(t) x(t-\tau)]^{\prime}+Q_{1}(t) x\left(t-\sigma_{1}\right)-Q_{2}(t) x\left(t-\sigma_{2}\right)=0, \quad \forall t \geq t_{0},
$$

where $p \in C\left(\left[t_{0},+\infty\right), \mathbb{R}\right)$. Liu et al. [2] got the existence of bounded nonoscillatory solutions for the first order nonlinear neutral delay differential equation

$$
\begin{aligned}
& {[x(t)+c(t) x(t-\tau)]^{\prime}+h(t) f\left(x\left(t-\sigma_{1}\right), x\left(t-\sigma_{2}\right), \ldots, x\left(t-\sigma_{k}\right)\right)} \\
& =g(t), \quad \forall t \geq t_{0},
\end{aligned}
$$

where $c \in C\left(\left[t_{0},+\infty\right), \mathbb{R}\right)$. Kulenović and Hadžiomerspahić [1] studied the existence of a nonoscillatory solution for the second order linear neutral delay differential equation with positive and negative coefficients

$$
[x(t)+p x(t-\tau)]^{\prime \prime}+Q_{1}(t) x\left(t-\sigma_{1}\right)-Q_{2}(t) x\left(t-\sigma_{2}\right)=0, \quad \forall t \geq t_{0},
$$

where $p \in \mathbb{R} \backslash\{ \pm 1\}$. Zhou [4] discussed solvability of the second order nonlinear neutral differential equation

$$
\left[r(t)(x(t)+p(t) x(t-\tau))^{\prime}\right]^{\prime}+\sum_{i=1}^{m} q_{i}(t) f_{i}\left(x\left(t-\sigma_{i}\right)\right)=0, \quad \forall t \geq t_{0},
$$

where $p \in C\left(\left[t_{0},+\infty\right), \mathbb{R}\right)$.

Motivated by the papers mentioned above, in this paper, we investigate the following second order neutral delay differential equation

$$
\begin{aligned}
& {\left[a(t)(x(t)-p(t) x(t-\tau))^{\prime}\right]^{\prime}+f\left(t, x\left(t-\alpha_{1}\right), x\left(t-\alpha_{2}\right), \ldots, x\left(t-\alpha_{k}\right)\right)} \\
& =g(t), \quad \forall t \geq t_{0},
\end{aligned}
$$

where $\tau>0, k \in \mathbb{N}, t_{0} \in \mathbb{R}, \alpha_{j} \in \mathbb{R}^{+}$for $j \in\{1,2, \ldots, k\}, p, g \in C\left(\left[t_{0},+\infty\right), \mathbb{R}\right)$, $a \in C\left(\left[t_{0},+\infty\right), \mathbb{R} \backslash\{0\}\right)$ and $f \in C\left(\left[t_{0},+\infty\right) \times \mathbb{R}^{k}, \mathbb{R}\right)$.

By applying the Banach fixed point theorem, we obtain the existence of uncountably many bounded positive solutions and convergence of the Mann iterative methods for Eq.(1.5), respectively. Three examples are included.

Throughout this paper, we assume that $\mathbb{R}=(-\infty,+\infty), \mathbb{R}^{+}=[0,+\infty)$,

$$
\beta=t_{0}-\max \left\{\tau, \alpha_{i}: i \in\{1,2, \ldots, k\}\right\},
$$


$B C([\beta,+\infty), \mathbb{R})$ is the Banach space of all continuous bounded functions on $[\beta,+\infty)$ with norm

$$
\|x\|=\sup _{t \geq \beta}|x(t)| \quad \text { for each } x \in B C([\beta,+\infty), \mathbb{R})
$$

and

$$
A(N, M)=\{x \in B C([\beta,+\infty), \mathbb{R}): N \leq x(t) \leq M, t \geq \beta\} \quad \text { for } M>N>0
$$

It is easy to see that $A(N, M)$ is a bounded closed and convex subset of $B C([\beta,+\infty), \mathbb{R})$.

By a solution of Eq.(1.5) we mean a function $x \in B C([\beta,+\infty), \mathbb{R})$ for some $T \geq \tau+\left|t_{0}\right|+|\beta|$, such that $x(t)-p(t) x(t-\tau)$ and $a(t)(x(t)-p(t) x(t-\tau))^{\prime}$ are continuously differentiable on $[T,+\infty)$ and Eq.(1.5) is satisfied for $t \geq T$.

\section{Existence of Uncountably Many Bounded Positive Solutions}

Now we use the Banach fixed point theorem to show the existence of uncountably many bounded positive solutions of Eq.(1.5).

Theorem 2.1. Assume that there exist constants $M, N, p_{0}$ and functions $q, r \in C\left(\left[t_{0},+\infty\right), \mathbb{R}^{+}\right)$satisfying

$$
\begin{gathered}
\left|f\left(t, u_{1}, u_{2}, \ldots, u_{k}\right)\right| \leq q(t), \\
\forall\left(t, u_{l}\right) \in\left[t_{0},+\infty\right) \times[N, M], l \in\{1,2, \ldots, k\} ; \\
\left|f\left(t, u_{1}, u_{2}, \ldots, u_{k}\right)-f\left(t, v_{1}, v_{2}, \ldots, v_{k}\right)\right| \\
\leq r(t) \max \left\{\left|u_{l}-v_{l}\right|: 1 \leq l \leq k\right\}, \\
\quad \forall\left(t, u_{l}, v_{l}\right) \in\left[t_{0},+\infty\right) \times[N, M]^{2}, l \in\{1,2, \ldots, k\} ; \\
\int_{t_{0}}^{+\infty} \frac{1}{|a(u)|} \int_{u}^{+\infty} \max \{q(s), r(s),|g(s)|\} d s d u<+\infty ; \\
0<N<\left(1-2 p_{0}\right) M, \quad|p(t)| \leq p_{0}<\frac{1}{2}, \quad \forall t \geq t_{0} .
\end{gathered}
$$

Then (a) for any $L \in\left(N+p_{0} M,\left(1-p_{0}\right) M\right)$, there exist $\theta \in(0,1)$ and $T>\tau+$ $\left|t_{0}\right|+|\beta|$ such that for each $x_{0} \in A(N, M)$, the Mann iterative sequence $\left\{x_{m}\right\}_{m \geq 0}$ 
generated by the scheme

$$
x_{m+1}(t)=\left\{\begin{array}{c}
\left(1-\alpha_{m}\right) x_{m}(t)+\alpha_{m}\left\{L+p(t) x_{m}(t-\tau)\right. \\
-\int_{t}^{+\infty} \frac{1}{a(u)} \int_{u}^{+\infty}\left[f \left(s, x_{m}\left(s-\alpha_{1}\right), x_{m}\left(s-\alpha_{2}\right),\right.\right. \\
\left.\left.\left.\ldots, x_{m}\left(s-\alpha_{k}\right)\right)-g(s)\right] d s d u\right\}, \quad \forall t \geq T, m \geq 0, \\
\left(1-\alpha_{m}\right) x_{m}(T)+\alpha_{m}\left\{L+p(T) x_{m}(T-\tau)\right. \\
-\int_{T}^{+\infty} \frac{1}{a(u)} \int_{u}^{+\infty}\left[f \left(s, x_{m}\left(s-\alpha_{1}\right), x_{m}\left(s-\alpha_{2}\right),\right.\right. \\
\left.\left.\left.\ldots, x_{m}\left(s-\alpha_{k}\right)\right)-g(s)\right] d s d u\right\}, \quad \forall \beta \leq t<T, m \geq 0
\end{array}\right.
$$

converges to a bounded positive solution $x \in A(N, M)$ of Eq.(1.5) and has the following error estimate:

$$
\left\|x_{m+1}-x\right\| \leq e^{-(1-\theta) \sum_{n=0}^{m} \alpha_{n}}\left\|x_{0}-x\right\|, \quad \forall m \geq 0
$$

where $\left\{\alpha_{m}\right\}_{m \geq 0}$ is an arbitrary sequence in $[0,1]$ such that

$$
\sum_{m=0}^{\infty} \alpha_{m}=+\infty
$$

(b) Eq.(1.5) possesses uncountably many bounded positive solutions in $A(N$, $M)$.

Proof. Firstly, we prove that (a) holds. Let $L \in\left(N+p_{0} M,\left(1-p_{0}\right) M\right)$. By means of (2.3) and (2.4), we deduce that there exist $\theta \in(0,1)$ and $T>$ $\tau+\left|t_{0}\right|+|\beta|$ satisfying

$$
\begin{aligned}
& \theta=\left|p_{0}\right|+\int_{T}^{+\infty} \frac{1}{|a(u)|} \int_{u}^{+\infty} r(s) d s d u \\
& \int_{T}^{+\infty} \frac{1}{|a(u)|} \int_{u}^{+\infty}[q(s)+|g(s)|] d s d u \\
& \leq \min \left\{\left(1-p_{0}\right) M-L, L-N-p_{0} M\right\} .
\end{aligned}
$$

Define a mapping $S_{L}: A(N, M) \rightarrow B C([\beta,+\infty), \mathbb{R})$ by

$$
S_{L} x(t)=\left\{\begin{array}{c}
L+p(t) x(t-\tau) \\
-\int_{t}^{+\infty} \frac{1}{a(u)} \int_{u}^{+\infty}\left[f \left(s, x\left(s-\alpha_{1}\right), x\left(s-\alpha_{2}\right),\right.\right. \\
\left.\left.\ldots, x\left(s-\alpha_{k}\right)\right)-g(s)\right] d s d u, \quad \forall t \geq T, x \in A(N, M), \\
S_{L} x(T), \quad \forall \beta \leq t<T, x \in A(N, M) .
\end{array}\right.
$$


Obviously, $S_{L} x$ is continuous for each $x \in A(N, M)$. From (2.2), (2.4), (2.8) and (2.10), we infer that for any $x, y \in A(N, M)$ and $t \geq T$

$$
\begin{aligned}
\left|S_{L} x(t)-S_{L} y(t)\right| & \mid L+p(t) x(t-\tau)-\int_{t}^{+\infty} \frac{1}{a(u)} \int_{u}^{+\infty}\left[f \left(s, x\left(s-\alpha_{1}\right), x\left(s-\alpha_{2}\right),\right.\right. \\
= & \left.\left.\ldots, x\left(s-\alpha_{k}\right)\right)-g(s)\right] d s d u \\
& \quad L-p(t) y(t-\tau)+\int_{t}^{+\infty} \frac{1}{a(u)} \int_{u}^{+\infty}\left[f \left(s, y\left(s-\alpha_{1}\right), y\left(s-\alpha_{2}\right),\right.\right. \\
& \left.\left.\quad \ldots, y\left(s-\alpha_{k}\right)\right)-g(s)\right] d s d u \mid \\
\leq & |p(t)||x(t-\tau)-y(t-\tau)| \\
& +\int_{t}^{+\infty} \frac{1}{|a(u)|} \int_{u}^{+\infty} \mid f\left(s, x\left(s-\alpha_{1}\right), x\left(s-\alpha_{2}\right), \ldots, x\left(s-\alpha_{k}\right)\right) \\
& \quad-f\left(s, y\left(s-\alpha_{1}\right), y\left(s-\alpha_{2}\right), \ldots, y\left(s-\alpha_{k}\right)\right) \mid d s d u \\
\leq & p_{0}\|x-y\| \\
& +\int_{t}^{+\infty} \frac{1}{|a(u)|} \int_{u}^{+\infty} r(s) \max \left\{\left|x\left(s-\alpha_{l}\right)-y\left(s-\alpha_{l}\right)\right|: 1 \leq l \leq k\right\} d s d u \\
\leq & p_{0}\|x-y\|+\|x-y\| \int_{t}^{+\infty} \frac{1}{|a(u)|} \int_{u}^{+\infty} r(s) d s d u \\
\leq & \left(p_{0}+\int_{T}^{+\infty} \frac{1}{|a(u)|} \int_{u}^{+\infty} r(s) d s d u\right)\|x-y\| \\
= & \theta\|x-y\|,
\end{aligned}
$$

which gives that

$$
\left\|S_{L} x-S_{L} y\right\| \leq \theta\|x-y\|, \quad \forall x, y \in A(N, M) .
$$

In view of (2.1), (2.4), (2.9) and (2.10), we conclude that for any $x \in A(N, M)$ and $t \geq T$

$$
\begin{aligned}
S_{L} x(t)= & L+p(t) x(t-\tau)-\int_{t}^{+\infty} \frac{1}{a(u)} \int_{u}^{+\infty}\left[f \left(s, x\left(s-\alpha_{1}\right), x\left(s-\alpha_{2}\right),\right.\right. \\
& \left.\left.\ldots, x\left(s-\alpha_{k}\right)\right)-g(s)\right] d s d u \\
\leq & L+|p(t)||x(t-\tau)|+\int_{t}^{+\infty} \frac{1}{|a(u)|} \int_{u}^{+\infty}\left[\mid f\left(s, x\left(s-\alpha_{1}\right), x\left(s-\alpha_{2}\right),\right.\right. \\
& \left.\left.\ldots, x\left(s-\alpha_{k}\right)\right)|+| g(s) \mid\right] d s d u \\
\leq & L+p_{0} M+\int_{t}^{+\infty} \frac{1}{|a(u)|} \int_{u}^{+\infty}[q(s)+|g(s)|] d s d u
\end{aligned}
$$




$$
\begin{aligned}
& \leq L+p_{0} M+\int_{T}^{+\infty} \frac{1}{|a(u)|} \int_{u}^{+\infty}[q(s)+|g(s)|] d s d u \\
& \leq L+p_{0} M+\min \left\{\left(1-p_{0}\right) M-L, L-N-p_{0} M\right\} \\
& \leq M
\end{aligned}
$$

and

$$
\begin{aligned}
S_{L} x(t)= & L+p(t) x(t-\tau)-\int_{t}^{+\infty} \frac{1}{a(u)} \int_{u}^{+\infty}\left[f \left(s, x\left(s-\alpha_{1}\right), x\left(s-\alpha_{2}\right),\right.\right. \\
& \left.\left.\ldots, x\left(s-\alpha_{k}\right)\right)-g(s)\right] d s d u \\
\geq & L-|p(t)||x(t-\tau)|-\int_{t}^{+\infty} \frac{1}{|a(u)|} \int_{u}^{+\infty}\left[\mid f\left(s, x\left(s-\alpha_{1}\right), x\left(s-\alpha_{2}\right),\right.\right. \\
& \left.\left.\quad \ldots, x\left(s-\alpha_{k}\right)\right)|+| g(s) \mid\right] d s d u \\
\geq & L-p_{0} M-\int_{t}^{+\infty} \frac{1}{|a(u)|} \int_{u}^{+\infty}[q(s)+|g(s)|] d s d u \\
\geq & L-p_{0} M-\int_{T}^{+\infty} \frac{1}{|a(u)|} \int_{u}^{+\infty}[q(s)+|g(s)|] d s d u \\
\geq & L-p_{0} M-\min \left\{\left(1-p_{0}\right) M-L, L-N-p_{0} M\right\} \\
\geq & N,
\end{aligned}
$$

which yield that $S_{L}(A(N, M)) \subseteq A(N, M)$. It follows from (2.11) that $S_{L}$ is a contraction mapping and it has a fixed point $x \in A(N, M)$, which is a bounded positive solution of Eq.(1.5). On account of (2.5), (2.10) and (2.11), we infer that for any $m \geq 0$ and $t \geq T$

$$
\begin{aligned}
\left|x_{m+1}(t)-x(t)\right|= & \mid\left(1-\alpha_{m}\right) x_{m}(t)+\alpha_{m}\left\{L+p(t) x_{m}(t-\tau)\right. \\
- & \int_{t}^{+\infty} \frac{1}{a(u)} \int_{u}^{+\infty}\left[f \left(s, x_{m}\left(s-\alpha_{1}\right), x_{m}\left(s-\alpha_{2}\right),\right.\right. \\
& \left.\left.\left.\quad \ldots, x_{m}\left(s-\alpha_{k}\right)\right)-g(s)\right] d s d u\right\}-x(t) \mid \\
\leq & \left(1-\alpha_{m}\right)\left|x_{m}(t)-x(t)\right|+\alpha_{m}\left|S_{L} x_{m}(t)-S_{L} x(t)\right| \\
\leq & \left(1-\alpha_{m}\right)\left\|x_{m}-x\right\|+\alpha_{m} \theta\left\|x_{m}-x\right\| \\
\leq & e^{-(1-\theta) \alpha_{m}}\left\|x_{m}-x\right\|
\end{aligned}
$$

which gives that

$$
\left\|x_{m+1}-x\right\| \leq e^{-(1-\theta) \alpha_{m}}\left\|x_{m}-x\right\| \leq e^{-(1-\theta) \sum_{n=0}^{m} \alpha_{n}}\left\|x_{0}-x\right\|, \quad \forall m \geq 0,
$$

that is, (2.6) holds. Thus (2.6) and (2.7) mean that $\lim _{m \rightarrow \infty} x_{m}=x$.

Next we show that (b) holds. Let $L_{1}, L_{2} \in\left(N+p_{0} M,\left(1-p_{0}\right) M\right)$ with $L_{1} \neq L_{2}$. As in the proof of (a), for each $i \in\{1,2\}$ there exist $\theta_{i} \in(0,1), T_{i}>$ 
$\tau+\left|t_{0}\right|+|\beta|$ and a mapping $S_{L_{i}}: A(N, M) \rightarrow B C([\beta,+\infty), \mathbb{R})$ satisfying (2.8)-(2.10), where $\theta, T, S_{L}$ are replaced by $\theta_{i}, T_{i}, S_{L_{i}}$, respectively, and $S_{L_{i}}$ has a fixed point $z_{i} \in A(N, M)$, which is also a bounded positive solution of Eq.(1.5), that is,

$$
\begin{aligned}
z_{1}(t)= & L_{1}+p(t) z_{1}(t-\tau) \\
- & \int_{t}^{+\infty} \frac{1}{a(u)} \int_{u}^{+\infty}\left[f \left(s, z_{1}\left(s-\alpha_{1}\right), z_{1}\left(s-\alpha_{2}\right),\right.\right. \\
& \left.\left.\quad \ldots, z_{1}\left(s-\alpha_{k}\right)\right)-g(s)\right] d s d u, \quad \forall t \geq T_{1}
\end{aligned}
$$

and

$$
\begin{aligned}
z_{2}(t)= & L_{2}+p(t) z_{2}(t-\tau) \\
- & \int_{t}^{+\infty} \frac{1}{a(u)} \int_{u}^{+\infty}\left[f \left(s, z_{2}\left(s-\alpha_{1}\right), z_{2}\left(s-\alpha_{2}\right),\right.\right. \\
& \left.\left.\quad \ldots, z_{2}\left(s-\alpha_{k}\right)\right)-g(s)\right] d s d u, \quad \forall t \geq T_{2} .
\end{aligned}
$$

In order to prove (b), we need only to show that $z_{1} \neq z_{2}$. In fact, (2.2), (2.4), (2.8), (2.12) and (2.13) give that for any $t \geq \max \left\{T_{1}, T_{2}\right\}$

$$
\begin{aligned}
& \left|z_{1}(t)-z_{2}(t)\right| \\
& =\mid L_{1}+p(t) z_{1}(t-\tau)-\int_{t}^{+\infty} \frac{1}{a(u)} \int_{u}^{+\infty}\left[f \left(s, z_{1}\left(s-\alpha_{1}\right), z_{1}\left(s-\alpha_{2}\right),\right.\right. \\
& \left.\left.\ldots, z_{1}\left(s-\alpha_{k}\right)\right)-g(s)\right] d s d u \\
& -L_{2}-p(t) z_{2}(t-\tau)+\int_{t}^{+\infty} \frac{1}{a(u)} \int_{u}^{+\infty}\left[f \left(s, z_{2}\left(s-\alpha_{1}\right), z_{2}\left(s-\alpha_{2}\right),\right.\right. \\
& \left.\left.\ldots, z_{2}\left(s-\alpha_{k}\right)\right)-g(s)\right] d s d u \\
& \geq\left|L_{1}-L_{2}\right|-|p(t)|\left|z_{1}(t-\tau)-z_{2}(t-\tau)\right| \\
& -\int_{t}^{+\infty} \frac{1}{|a(u)|} \int_{u}^{+\infty} \mid f\left(s, z_{1}\left(s-\alpha_{1}\right), z_{1}\left(s-\alpha_{2}\right), \ldots, z_{1}\left(s-\alpha_{k}\right)\right) \\
& -f\left(s, z_{2}\left(s-\alpha_{1}\right), z_{2}\left(s-\alpha_{2}\right), \ldots, z_{2}\left(s-\alpha_{k}\right)\right) \mid d s d u \\
& \geq\left|L_{1}-L_{2}\right|-p_{0}\left\|z_{1}-z_{2}\right\|-\left\|z_{1}-z_{2}\right\| \int_{t}^{+\infty} \frac{1}{|a(u)|} \int_{u}^{+\infty} r(s) d s d u \\
& \geq\left|L_{1}-L_{2}\right|-\left\|z_{1}-z_{2}\right\|\left(p_{0}+\int_{t}^{+\infty} \frac{1}{|a(u)|} \int_{u}^{+\infty} r(s) d s d u\right) \\
& \geq\left|L_{1}-L_{2}\right|-\left\|z_{1}-z_{2}\right\|\left(p_{0}+\int_{\max \left\{T_{1}, T_{2}\right\}}^{+\infty} \frac{1}{|a(u)|} \int_{u}^{+\infty} r(s) d s d u\right) \\
& \geq\left|L_{1}-L_{2}\right|-\left\|z_{1}-z_{2}\right\| \max \left\{\theta_{1}, \theta_{2}\right\},
\end{aligned}
$$

which yields that

$$
\left\|z_{1}-z_{2}\right\| \geq \frac{\left|L_{1}-L_{2}\right|}{1+\max \left\{\theta_{1}, \theta_{2}\right\}}>0
$$


that is, $z_{1} \neq z_{2}$. This completes the proof.

Theorem 2.2. Assume that there exist constants $M, N, p_{0}$ and functions $q, r \in C\left(\left[t_{0},+\infty\right), \mathbb{R}^{+}\right)$satisfying $(2.1)-(2.3)$ and

$$
0<N<\left(1-p_{0}\right) M, \quad 0 \leq p(t) \leq p_{0}<1, \quad \forall t \geq t_{0} .
$$

Then (a) for each $L \in\left(N,\left(1-p_{0}\right) M\right)$, there exist $\theta \in(0,1)$ and $T>\tau+\left|t_{0}\right|+$ $|\beta|$ such that for each $x_{0} \in A(N, M)$, the Mann iterative sequence $\left\{x_{m}\right\}_{m \geq 0}$ generated by (2.5) with (2.7) converges to a bounded positive solution $x \in$ $A(N, M)$ of Eq.(1.5) and has the error estimate (2.6);

(b) Eq.(1.5) has uncountably many bounded positive solutions in $A(N, M)$.

Proof. Let $L \in\left(N,\left(1-p_{0}\right) M\right)$. By virtue of (2.3) and (2.14), we get that there exist $\theta \in(0,1)$ and $T>\tau+\left|t_{0}\right|+|\beta|$ satisfying (2.8) and

$$
\int_{T}^{+\infty} \frac{1}{|a(u)|} \int_{u}^{+\infty}[q(s)+|g(s)|] d s d u \leq \min \left\{\left(1-p_{0}\right) M-L, L-N\right\} .
$$

Let the mapping $S_{L}: A(N, M) \rightarrow B C([\beta,+\infty), \mathbb{R})$ be defined by $(2.10)$. Combining (2.1), (2.10), (2.14) and (2.15), we derive that for any $x, y \in A(N, M)$ and $t \geq T$

$$
\begin{aligned}
S_{L} x(t)= & L+p(t) x(t-\tau)-\int_{t}^{+\infty} \frac{1}{a(u)} \int_{u}^{+\infty}\left[f \left(s, x\left(s-\alpha_{1}\right), x\left(s-\alpha_{2}\right),\right.\right. \\
& \left.\left.\ldots, x\left(s-\alpha_{k}\right)\right)-g(s)\right] d s d u \\
\leq & L+|p(t)||x(t-\tau)|+\int_{t}^{+\infty} \frac{1}{|a(u)|} \int_{u}^{+\infty}\left[\mid f\left(s, x\left(s-\alpha_{1}\right), x\left(s-\alpha_{2}\right),\right.\right. \\
& \left.\left.\ldots, x\left(s-\alpha_{k}\right)\right)|+| g(s) \mid\right] d s d u \\
\leq & L+p_{0} M+\int_{t}^{+\infty} \frac{1}{|a(u)|} \int_{u}^{+\infty}[q(s)+|g(s)|] d s d u \\
\leq & L+p_{0} M+\int_{T}^{+\infty} \frac{1}{|a(u)|} \int_{u}^{+\infty}[q(s)+|g(s)|] d s d u \\
\leq & L+p_{0} M+\min \left\{\left(1-p_{0}\right) M-L, L-N\right\} \\
\leq & M
\end{aligned}
$$

and

$$
\begin{gathered}
S_{L} x(t)=L+p(t) x(t-\tau)-\int_{t}^{+\infty} \frac{1}{a(u)} \int_{u}^{+\infty}\left[f \left(s, x\left(s-\alpha_{1}\right), x\left(s-\alpha_{2}\right),\right.\right. \\
\left.\left.\ldots, x\left(s-\alpha_{k}\right)\right)-g(s)\right] d s d u \\
\geq L-\int_{t}^{+\infty} \frac{1}{|a(u)|} \int_{u}^{+\infty}\left[\mid f\left(s, x\left(s-\alpha_{1}\right), x\left(s-\alpha_{2}\right),\right.\right. \\
\left.\left.\ldots, x\left(s-\alpha_{k}\right)\right)|+| g(s) \mid\right] d s d u
\end{gathered}
$$




$$
\begin{aligned}
& \geq L-\int_{t}^{+\infty} \frac{1}{|a(u)|} \int_{u}^{+\infty}[q(s)+|g(s)|] d s d u \\
& \geq L-\int_{T}^{+\infty} \frac{1}{|a(u)|} \int_{u}^{+\infty}[q(s)+|g(s)|] d s d u \\
& \geq L-\min \left\{\left(1-p_{0}\right) M-L, L-N\right\} \\
& \geq N,
\end{aligned}
$$

which mean that $S_{L}(A(N, M)) \subseteq A(N, M)$. The rest of the proof is similar to that of Theorem 2.1, and is omitted. This completes the proof.

Theorem 2.3. Assume that there exist constants $M, N, p_{0}$ and functions $q, r \in C\left(\left[t_{0},+\infty\right), \mathbb{R}^{+}\right)$satisfying $(2.1)-(2.3)$ and

$$
0<N<\left(1+p_{0}\right) M, \quad-1<p_{0} \leq p(t) \leq 0, \quad \forall t \geq t_{0}
$$

Then (a) for each $L \in\left(N-p_{0} M, M\right)$, there exist $\theta \in(0,1)$ and $T>\tau+\left|t_{0}\right|+$ $|\beta|$ such that for each $x_{0} \in A(N, M)$, the Mann iterative sequence $\left\{x_{m}\right\}_{m \geq 0}$ generated by (2.5) with (2.7) converges to a bounded positive solution $x \in$ $A(N, M)$ of Eq.(1.5) and has the error estimate (2.6);

(b) Eq.(1.5) has uncountably many bounded positive solutions in $A(N, M)$.

Proof. Let $L \in\left(N-p_{0} M, M\right)$. It follows from (2.3) and (2.16) that there exist $\theta \in(0,1)$ and $T>\tau+\left|t_{0}\right|+|\beta|$ satisfying (2.8) and

$$
\int_{T}^{+\infty} \frac{1}{|a(u)|} \int_{u}^{+\infty}[q(s)+|g(s)|] d s d u \leq \min \left\{M-L, L-N+p_{0} M\right\} .
$$

Let the mapping $S_{L}: A(N, M) \rightarrow B C([\beta,+\infty), \mathbb{R})$ be defined by $(2.10)$. In light of (2.1), (2.10), (2.16) and (2.17), we know that for any $x, y \in A(N, M)$ and $t \geq T$

$$
\begin{aligned}
S_{L} x(t)= & L+p(t) x(t-\tau)-\int_{t}^{+\infty} \frac{1}{a(u)} \int_{u}^{+\infty}\left[f \left(s, x\left(s-\alpha_{1}\right), x\left(s-\alpha_{2}\right),\right.\right. \\
& \left.\left.\ldots, x\left(s-\alpha_{k}\right)\right)-g(s)\right] d s d u \\
\leq & L+\int_{t}^{+\infty} \frac{1}{|a(u)|} \int_{u}^{+\infty}\left[\mid f\left(s, x\left(s-\alpha_{1}\right), x\left(s-\alpha_{2}\right),\right.\right. \\
& \left.\left.\quad \ldots, x\left(s-\alpha_{k}\right)\right)|+| g(s)\right] d s d u \\
\leq & L+\int_{t}^{+\infty} \frac{1}{|a(u)|} \int_{u}^{+\infty}[q(s)+|g(s)|] d s d u \\
\leq & L+\int_{T}^{+\infty} \frac{1}{|a(u)|} \int_{u}^{+\infty}[q(s)+|g(s)|] d s d u \\
\leq & L+\min \left\{M-L, L-N+{ }_{0} p M\right\} \\
\leq & M
\end{aligned}
$$


and

$$
\begin{aligned}
S_{L} x(t)= & L+p(t) x(t-\tau)-\int_{t}^{+\infty} \frac{1}{a(u)} \int_{u}^{+\infty}\left[f \left(s, x\left(s-\alpha_{1}\right), x\left(s-\alpha_{2}\right),\right.\right. \\
& \left.\left.\ldots, x\left(s-\alpha_{k}\right)\right)-g(s)\right] d s d u \\
\geq & L+p_{0} M-\int_{t}^{+\infty} \frac{1}{|a(u)|} \int_{u}^{+\infty}\left[\mid f\left(s, x\left(s-\alpha_{1}\right), x\left(s-\alpha_{2}\right),\right.\right. \\
& \left.\left.\ldots, x\left(s-\alpha_{k}\right)\right)|+| g(s) \mid\right] d s d u \\
\geq & L+p_{0} M-\int_{t}^{+\infty} \frac{1}{|a(u)|} \int_{u}^{+\infty}[q(s)+|g(s)|] d s d u \\
\geq & L+p_{0} M-\int_{T}^{+\infty} \frac{1}{|a(u)|} \int_{u}^{+\infty}[q(s)+|g(s)|] d s d u \\
\geq & L+p_{0} M-\min \left\{M-L, L-N+p_{0} M\right\} \\
\geq & N,
\end{aligned}
$$

which give that $S_{L}(A(N, M)) \subseteq A(N, M)$. The rest of the proof is similar to that of Theorem 2.1, and is omitted. This completes the proof.

\section{Examples and Applications}

Now we construct three examples as applications of the results presented in Section 2.

Example 3.1. Consider the following second order neutral delay differential equation

$$
\begin{gathered}
{\left[\left(2 t^{6}+t \ln ^{2} t-1\right)\left(x(t)-\frac{t^{2} \sin \left(t+\sqrt{t^{2}+1}+2\right)}{2+3 t^{2}} x(t-\tau)\right)^{\prime}\right]^{\prime}} \\
+\frac{\ln \left(1+t x^{2}(t-1)\right)}{t^{4}+\sin ^{2}(t-\sqrt{t-1})}=\frac{t^{2} \cos ^{2}\left(t^{2}-4 t+1\right)}{\left(1+t^{2}\right)^{5}}, \quad \forall t \geq 1,
\end{gathered}
$$

where $\tau>0$. Let $t_{0}=1, k=1, \alpha_{1}=1, p_{0}=\frac{1}{3}, N=1, M=4, \beta=$ $1-\max \{\tau, 1\}$ and $\forall(t, u) \in\left[t_{0},+\infty\right) \times \mathbb{R}$

$$
\begin{aligned}
& a(t)=2 t^{6}+t \ln ^{2} t-1, \quad p(t)=\frac{t^{2} \sin \left(t+\sqrt{t^{2}+1}+2\right)}{2+3 t^{2}}, \\
& g(t)=\frac{t^{2} \cos ^{2}\left(t^{2}-4 t+1\right)}{\left(1+t^{2}\right)^{5}}, \quad f(t, u)=\frac{\ln \left(1+t u^{2}\right)}{t^{4}+\sin ^{2}(t-\sqrt{t-1})}, \\
& q(t)=\frac{\ln \left(1+t M^{2}\right)}{t^{4}+\sin ^{2}(t-\sqrt{t-1})}, \quad r(t)=\frac{2 M t}{t^{4}+\sin ^{2}(t-\sqrt{t-1})} .
\end{aligned}
$$


It is easy to verify that (2.1)-(2.4) are satisfied. It follows from Theorem 2.1 that Eq.(3.1) possesses uncountably many bounded positive solutions in $A(N, M)$. On the other hand, for any $L \in\left(N+p_{0} M,\left(1-p_{0}\right) M\right)$, there exist $\theta \in(0,1)$ and $T \geq \tau+\left|t_{0}\right|+|\beta|$ such that the Mann iterative sequence $\left\{x_{m}\right\}_{m \geq 0}$ generated by (2.5) with (2.7) converges to a bounded positive solution $x \in$ $A(N, M)$ of Eq.(3.1) and has the error estimate (2.6).

Example 3.2. Consider the second order neutral delay differential equation

$$
\begin{aligned}
& {\left[\left(t^{3}+2 t^{2}-t\right)\left(x(t)-\frac{(3+\sin t) t^{4}}{1+5 t^{4}} x(t-\tau)\right)^{\prime}\right]^{\prime}} \\
& \quad+\frac{t^{3} x^{3}(t-3)-x^{2}(t-7)}{t^{15}+x^{2}(t-2)}=\frac{\sin \left(t^{2}\right)}{t^{6}}, \quad \forall t \geq 2
\end{aligned}
$$

where $\tau>0$. Let $t_{0}=2, k=3, \alpha_{1}=3, \alpha_{2}=7, \alpha_{3}=2, p_{0}=\frac{4}{5}, N=10$, $M=100, \beta=2-\max \{\tau, 7\}$ and $\forall(t, u, v, w) \in\left[t_{0},+\infty\right) \times \mathbb{R}^{3}$

$$
\begin{aligned}
& a(t)=t^{3}+2 t^{2}-t, \quad p(t)=\frac{(3+\sin t) t^{4}}{1+5 t^{4}}, \quad g(t)=\frac{\sin \left(t^{2}\right)}{t^{6}}, \\
& f(t, u, v, w)=\frac{t^{3} u^{3}-v^{2}}{t^{15}+w^{2}}, \quad q(t)=\frac{M^{3} t^{3}+M^{2}}{t^{15}+N^{2}} \\
& r(t)=\frac{3 M^{2} t^{18}+2 M t^{15}+8 M^{4} t^{3}+4 M^{3}}{\left(N^{2}+t^{15}\right)^{2}} .
\end{aligned}
$$

It is easy to verify that (2.1)-(2.3) and (2.14) are satisfied. It follows from Theorem 2.2 that Eq.(3.2) possesses uncountably many bounded positive solutions in $A(N, M)$. On the other hand, for any $L \in\left(N,\left(1-p_{0}\right) M\right)$, there exist $\theta \in(0,1)$ and $T \geq \tau+\left|t_{0}\right|+|\beta|$ such that the Mann iterative sequence $\left\{x_{m}\right\}_{m \geq 0}$ generated by (2.5) with (2.7) converges to a bounded positive solution $x \in A(N, M)$ of Eq.(3.2) and has the error estimate (2.6).

Example 3.3. Consider the second order neutral delay differential equation

$$
\begin{aligned}
& {\left[\left(t^{2} \ln ^{3} t+1\right)\left(x(t)+\frac{1+4 t+\cos ^{2} t}{2+7 t} x(t-\tau)\right)^{\prime}\right]^{\prime}} \\
& \quad+\frac{x^{3}(t-10)}{2 t^{3}-1}-\frac{t x^{2}(t-15)}{t^{4}+2 t-1}+\frac{x^{2}(t-10)}{t^{2}+x^{4}(t-20)} \\
& =\frac{1+\sqrt{t}-t^{8} \sin ^{5} t}{1+t^{21}}, \quad \forall t \geq 3,
\end{aligned}
$$

where $\tau>0$. Let $t_{0}=3, k=3, \alpha_{1}=10, \alpha_{2}=15, \alpha_{3}=20, p_{0}=-\frac{5}{7}, N=19$, 


$$
\begin{aligned}
& M=70, \beta=3-\max \{\tau, 20\} \text { and } \forall(t, u, v, w) \in\left[t_{0},+\infty\right) \times \mathbb{R}^{3} \\
& a(t)=t^{2} \ln ^{3} t+1, \quad p(t)=-\frac{1+4 t+\cos ^{2} t}{2+7 t}, \quad g(t)=\frac{1+\sqrt{t}-t^{8} \sin ^{5} t}{1+t^{21}} \\
& f(t, u, v, w)=\frac{u^{3}}{2 t^{3}-1}-\frac{t v^{2}}{t^{4}+2 t-1}+\frac{u^{2}}{t^{2}+w^{4}} \\
& q(t)=\frac{M^{3}}{2 t^{3}-1}+\frac{t M^{2}}{t^{4}+2 t-1}+\frac{M^{2}}{t^{2}+N^{4}} \\
& r(t)=\frac{3 M^{2}}{2 t^{3}-1}+\frac{2 M t}{t^{4}+2 t-1}+\frac{2 M t^{2}+6 M^{5}}{\left(t^{2}+N^{4}\right)^{2}}
\end{aligned}
$$

It is easy to verify that (2.1)-(2.3) and (2.16) are satisfied. It follows from Theorem 2.3 that Eq.(3.3) possesses uncountably many bounded positive solutions in $A(N, M)$. On the other hand, for any $L \in\left(N-p_{0} M, M\right)$, there exist $\theta \in(0,1)$ and $T \geq \tau+\left|t_{0}\right|+|\beta|$ such that the Mann iterative sequence $\left\{x_{m}\right\}_{m \geq 0}$ generated by (2.5) with (2.7) converges to a bounded positive solution $x \in A(N, M)$ of Eq.(3.3) and has the error estimate (2.6).

Acknowledgements. This research was supported by the Science Research Foundation of Educational Department of Liaoning Province (L2012380).

\section{References}

[1] M. R.S. Kulenović and S. Hadžiomerspahić, Existence of nonoscillatory solution of second order linear neutral delay equation, J. Math. Anal. Appl. 228 (1998), 436-448.

[2] Z. Liu, S. M. Kang and J.S. Ume, Existence of bounded nonoscillatory solutions of first-order nonlinear neutral delay differential equations, Comput. Math. Appl. 59 (2010), 3535-3547.

[3] W. P. Zhang, W. Feng, J. R. Yan and J. S. Song, Existence of nonoscillatory solutions of first-order neutral delay differential equations, Comput. Math. Appl. 49 (2005), 1021-1027.

[4] Y. Zhou, Existence of nonoscillatory solutions of second-order nonlinear delay differential equations, J. Math. Anal. Appl. 331 (2007), 91-96.

\section{Received: August, 2012}

